\title{
EDITOR'S COMMENT
}

The November - December 2003 issue of the International Braz J Urol presents interesting contributions and as usual the Editor's Comment will be close to the list of contents and will highlight some important papers.

Doctor Braga and co-workers, from Charles Nicolle University Hospital, Rouen, France, compared on page 528 the characteristics of prenatally and postnatally diagnosed ureteropelvic junction obstruction (UPJO) in children. Of the 74 children evaluated, 44 (59.4\%) had a prenatal diagnosis of UPJO and $30(40.6 \%)$ had a postnatal diagnosis despite the fact that all had had a fetal ultrasonography. Clinical manifestations in children with postnatal UPJO included abdominal pain in $13(43 \%)$ patients, pyelonephritis in $7(23 \%)$, urinary tract infection in $5(16.6 \%)$, and occasional findings upon ultrasound in $5(16.6 \%)$. The authors concluded that postnatally diagnosed UPJO might be considered, at least in part, an entity different from prenatally detected obstruction due to its peculiar characteristics, i.e., postnatal UPJO more frequently affects females, manifests later in life with urinary infection or abdominal pain, and is frequently associated with ureteral kinking.

Doctors Abreu and Gill, from Glickman Urological Institute, Cleveland Clinic Foundation, Cleveland, Ohio, USA, world-recognized experts in the field, provided our readers on page 489 with a thorough description on the critical steps of the laparoscopic radical prostatectomy (LRP) technique. Also, the authors discussed how they impact upon the pertinent issues regarding prostate cancer surgery, that is, blood loss, potency and continence. The authors found based on their experience, that a major advantage of LRP is the reduced operative blood loss. At the Cleveland Clinic, the average blood loss of their first 100 patients was $322.5 \mathrm{~mL}$, resulting in low transfusion rates (2\%), which is in contrast to open prostatectomy series with reported blood losses between $500 \mathrm{~mL}$ to $1000 \mathrm{~mL}$. The authors concluded based on the initial experience, that the laparoscopic approach may represent a reliable less invasive alternative to the conventional open approach

Doctor Leite and colleagues, from Syrian Lebanese Hospital, São Paulo, Brazil, assessed on page 497 the importance of quantifying the adenocarcinoma in prostate biopsies when determining the tumor's final stage in patients who undergo radical prostatectomy. Also, the authors tried to identify the best method for obtaining such data. An average of 12 fragments was evaluated per patient. In the multivariate analysis, the Gleason histological grade and the total percentage of tumor were strongly correlated with the neoplasia's final stage. The risk of the tumor not being confined was 3 for Gleason 7 tumors and 10.6 for Gleason 8 tumors or above. The authors concluded that when associated to the Gleason histological grade, tumor quantification in prostate biopsies is an important factor for determining organ-confined disease, and among the methods, total percentage of tumor is the most informative one. 
Doctor Arruda and co-workers, from Federal University of São Paulo, Brazil, presented on page 502 a very original work on the impact of prior prostatic surgery for benign prostatic hyperplasia (transurethral resection of the prostate or open prostatectomy) on urinary continence in patients submitted to radical cystectomy and orthotopic ileal neobladder. It was also analyzed the patient's age and the preservation of prostatic apex as influencing factors. The authors found that the only statistically significant factor that impacted upon urinary continence was previous prostatic surgery, with respectively $33 \%$ versus $7 \%$ rate of incontinence for patients previously operated on and for those without previous operation. Patient's age and preservation of prostate apex did not affect the continence rate. Doctors Ralph W. de Vere White, from University of California Davis Cancer Center, and Paul F. Schellhammer from Eastern Virginia Medical School, USA provided editorial comments on this article.

Doctor Sanches and colleagues from Campinas State University, S<o Paulo, Brazil, evaluated on page 507 the overall accuracy of renal scintigraphy with ${ }^{99 m}$ Tc-DTPA in the diagnosis of acute renal graft complications. The scintigraphic results were concordant with the biopsies in $86 \%$ of the cases studied. The sensitivities of renal scintigraphy for detection of acute tubular necrosis, acute rejection and cortical necrosis were $98 \%, 87 \%$ and $100 \%$, respectively. The authors concluded that renal scintigraphy ${ }^{99 \mathrm{~m}}$ Tc-DTPA showed a good overall accuracy in the detection of acute renal graft complications.

Finally, it is my pleasure to announce in this last issue of 2003, that the International Braz $J$ Urol continues to grow in acceptance and circulation. In addition to the 6,000 copies of the printed version of our Journal, which reach more than 60 countries, during the last 6 months, the electronic version has been receiving around 10,000 to 12,000 visits on-line every month, from 90 to 98 different countries. These figures include the International Braz J Urol among the most read urological journals. This success is thanks to the effort of our authors, ad-hoc reviewers, and consulting editors, which dedicated a considerable fraction of their time to our Journal, and I would like to express my sincere recognition for it. 